\title{
Review of: "Oculomotor corollary discharge signaling is related to repetitive behavior in children with autism spectrum disorder"
}

Tanu Wadhera

Potential competing interests: The author(s) declared that no potential competing interests exist.

The paper utilized multilevel modeling to examine an imapct of saccade landing site upon trans-saccadic perceptual judgements. The finding is practically viable showing that compared to TD participants, the children with ASD show sensitivity to target displacement and less reliance upon saccade landing site when spatial uncertainty of the post-saccadic target was high. The relationship between altered CD signaling and core ASD symptoms is of utmost importance and very attractive for the readers and researchers working in the domain of Autism and Neurodevelopmental disorders. 